\title{
ANÁLISIS DE LA RELACIÓN \\ ENTRE LAS VARIABLES DE ENTRENAMIENTO EN UN EQUIPO DE BALONCESTO
}

\author{
ANALYSIS OF THE RELATIONSHIP BETWEEN TRAINING \\ VARIABLES IN A BASKETBALL TEAM
}

\author{
ANÁLISE DA RELAÇÃO ENTRE AS VARIÁVEIS \\ DE TREINAMENTO EM UM TIME DE BASQUETE
}

\author{
Enrique Godoy ${ }^{1}$ \\ Sebastián $\mathrm{Feu}^{2}$ \\ Larissa Galatti ${ }^{3}$ \\ Sergio José Ibáñez ${ }^{4}$
}

1 Licenciado en Ciencias del Deporte. Grupo de Optimizaci n del Entrenamiento y Rendimiento Deportivo. Facultad de Ciencias del Deporte. Universidad de Extremadura. Espa a. E-mail: egodoyhe@alumnos.unex.es

2 Doctor en Ciencias del Deporte. Grupo de Optimizaci n del Entrenamiento y Rendimiento Deportivo. Facultad de Ciencias de la Educaci n. Universidad de Extremadura. Espa a. L nea de Investigaci n: E-mail: sfeu@unex.es

3 Doctora en Educaci n F sica. Pedagog a del Deporte. Faculdade de Ci ncias Aplicadas. Universidade Estadual de Campinas. Brasil E-mail: larissa.galatti@ fca.unicamp.br

4 Doctor en Educaci n F sica. Grupo de Optimizaci n del Entrenamiento y Rendimiento Deportivo. Facultad de Ciencias del Deporte. Universidad de Extremadura. Espa a. E-mail: sibanez@unex.es

Godoy, E.; Feu, S.; Galatti, L. \& Ib ez, S. J. (2017). An lisis de la relaci n entre las variables de entrenamiento en un equipo de baloncesto. Educaci $n$ F sica y Deporte, 36(1), 67-99, Ene.-Jun. http://doi.org/10.17533/udea.efyd.v36n1a05

10.17533/udea.efyd.v36n1a05

URL DOI: http://doi.org/10.17533/udea.efyd.v36n1a05

Art culos de investigaci $n$ 


\section{RESUMEN}

La selecci $\mathrm{n}$ y organizaci $\mathrm{n}$ de las tareas puede condicionar la carga de entrenamiento en baloncesto. Objetivo: analizar la relaci $\mathrm{n}$ entre Variables Pedag gicas (situaci $n$ de juego y nivel de oposici n), Organizativas y de Carga Externa. $\mathbf{M}$ todo: se analizaron 38 entrenamientos, compuestos por 340 tareas de un equipo de categor a cadete durante la temporada 2015/2016. Se realiz un doble filtrado de datos para eliminar las tareas de calentamiento y tiros libres. Se obtuvieron resultados descriptivos de todas las variables y se utilizaron los coeficientes de correlaci $\mathrm{n}$ de Pearson y Spearman para identificar las relaciones entre las variables. Resultados: se estableci una relaci $\mathrm{n}$ significativa entre las variables que definen la organizaci n del entrenamiento y las variables de carga externa. Las tareas en las que se agrupan pocos jugadores, con oposici n activa, utilizando espacios reducidos, con alta participaci $n$ y elevados niveles de tiempo de pr ctica motriz, incrementan la carga del entrenamiento.

PALABRAS CLAVE: Tarea, Entrenamiento, Variables Pedag gicas, Variables Carga externa, Variables Organizativas

\section{ABSTRACT}

Problem: The selection and organization of tasks may condition the training load. Objective: To analyze the relationship between the Pedagogical (g ame s ituation and o pposition I evel), Organizational, and External Load Variables. Method: A total of 38 trainings, composed of 340 tasks of a c adet category during the 2015/2016 season, were analyzed, and a double data filtering to eliminate warm-up and free-throw tasks was performed. escriptive results of all variables were obtained. Pearson's and Spearman's correlation coefficients were used to identify the relationships between the variables. Results: A significant relationship between the variables that define the training organization and the external load variables was established. The tasks in which few players are grouped, with active opposition, using reduced spaces, with high participation and high levels of motor practice time increase the training load.

KEYWORDS: Task, training, pedagogical variables, external load variables, organizational variables

\section{RESUMO}

A seleç o e organizaç o das tarefas podem influenciar a carga de treinamento. O objetivo analisar a relaç o entre as Vari veis Pedag gicas Situaç o de Jogo e N vel de Oposiç o e as Vari veis Organizacionais e as Vari veis de Carga Externa. Um total de 38 treinamentos foram analisados, 
compostos por 340 tarefas de um time de uma categoria sub 16 durante a temporada 2015/2016. Uma dupla filtragem de dados tamb m foi realizada para excluir as tarefas de aquecimento e lances livres. Resultados descritivos de todas as vari veis foram obtidos no estudo. Coeficientes de correlaç o de Pearson e Spearman foram utilizados para identificar relaç es entre as vari veis. Os resultados mostram a exist ncia de uma relaç o significativa entre as vari veis que definem a organizaç o da treinamento e Vari veis de Carga Externa. As tarefas em que poucos jogadores s o agrupados, com oposiç o ativa, utilizando espaços reduzidos, com alta participaç o e altos $\mathrm{n}$ veis de tempo de pr tica motora aumentam a carga de treinamento

PALAVRAS CHAVE: Tarefa, Treinamento, Vari veis Pedag gicas, Vari veis de Carga Externa, Vari veis Organizacionais 


\section{INTRODUCCIÓN ${ }^{1}$}

Los deportes colectivos son aquellos en los que la acci n de juego es el resultante de la interrelaci n entre participantes (Hern ndez, 1994). Esta interacci n es producida de manera simul$\mathrm{t}$ nea, donde un equipo coopera entre s para oponerse a otro, que act a tambi $n$ en cooperaci $n$ y que a su vez se opone al anterior. En baloncesto se suceden situaciones de juego complejas y diferentes, que dif cilmente se van a repetir, demandando del jugador una actividad perceptiva y decisional necesaria para adaptarse al entorno cambiante (Hoyos, 2014). Ferreira, lb ez \& Sampaio (2009) afirman que el baloncesto es un claro ejemplo de deporte que ha evolucionado a trav s de la incorporaci $n$ y modificaci $n$ reglamentaria desde sus or genes hasta la actualidad. Hoy es uno de los deportes de invasi n m s practicado a nivel mundial y que $m$ s repercusi $n$ tiene en los medios de comunicaci $\mathrm{n}$.

$M$ Itiples factores afectan el rendimiento en baloncesto. Seg n S nchez (2007), los aspectos antropom tricos, t cnico$\mathrm{t}$ cticos y la condici $\mathrm{n} f$ sica no parecen ser tan determinantes en el juego, pues en la actualidad la preparaci $n$ de estos aspectos en los diferentes equipos es muy similar. Otras variables tienen gran repercusi $\mathrm{n}$ en los resultados de los equipos de baloncesto, como el grado de interacci n socio afectiva entre los componentes del grupo deportivo, el apoyo econ mico-social, o el perfil psicol gico de los entrenadores y los jugadores.

Un campo en el que se investiga actualmente es en el control de la carga de entrenamiento (Impellizzeri, Rampinini, Coutts, Sassi \& Marcora, 2004; Castagna, Impellizzeri, Chaouachi, Bordon \& Manzi, 2011). Existen diferentes formas de conocer cu I

1 Este trabajo ha sido parcialmente subvencionado por la Ayuda a los Grupos de Investigaci n (GR18170) del Gobierno de Extremadura (Consejer a de Empleo, Empresa e Innovaci n); con la aportaci $n$ de la Uni $n$ Europea a trav s de los Fondos Europeos de Desarrollo Regional. 
es la carga administrada a los deportistas. Para ello se utilizan pa$r$ metros o ndices internos del organismo (carga interna) como la frecuencia cardiaca (FC) (Dehesa, Vaquera, Garc a \& Bay n, 2015; Sampaio, Abrantes \& Leite, 2009), el consumo de ox geno (VO2) (Akubat \& Abt, 2011) o la concentraci n de cido I ctico (LAC) (Coutts, Rampinini, Mancora, Castagna \& Impellizzeri, 2008), e ndices externos (carga externa) que muestran c mo y cu nto ha trabajado un deportista, como los kil metros recorridos, la velocidad de desplazamiento o el $\mathrm{n}$ mero de lanzamientos realizados en un partido o entrenamiento. Para Coque $(2008,2009)$ es necesaria la cuantificaci $n$ de la carga total del entrenamiento en baloncesto, entendida sta como la suma total de est mulos a los que el jugador se ve sometido durante el proceso de preparaci n o entrenamiento.

La determinaci $\mathrm{n}$ de la intensidad del ejercicio a trav $\mathrm{s}$ de la frecuencia cardiaca ha sido empleada como indicador de carga interna. Esta t cnica se encuentra muy desarrollada, con numerosos estudios de validaci $n$, se acepta universalmente y se utiliza en diferentes modalidades deportivas para establecer la carga del entrenamiento (Coutinho et al., 2016; G mez, Gamonales, Pino \& Ib ez, 2018; Pascual, Llorca, Carbonell \& P rez, 2016). A la hora de valorar los efectos que dicha carga produce en el organismo, algunos preparadores y deportistas utilizan la estimaci $\mathrm{n}$ de la intensidad realizada por el propio deportista. Se sabe, por ejemplo, que el uso de $m$ todos basados en la medici $n$ de la frecuencia cardiaca en deportes intermitentes puede subestimar los niveles de $\mathrm{m}$ xima intensidad, debido a una marcada dependencia del metabolismo anaer bico (Alexiou \& Coutts, 2008; Akubat \& Abt, 2011; Casamichana, Castellano, Calleja, San Rom n \& Castagna, 2013; Scott, Lockie, Knight, Clark \& Janse, 2013).

El control de la carga de entrenamiento en deportes colectivos ha sido, y es, una de las principales preocupaciones que los $\mathrm{t}$ cnicos tienen a la hora de dise ar la planificaci $\mathrm{n}$ de una tem- 
porada. Un buen ajuste de la carga de trabajo deber a garantizar la consecuci $\mathrm{n}$ de los objetivos planteados por el equipo $\mathrm{t}$ cnico (Tapia \& Hern ndez, 2011). El control de la carga de entrenamiento se puede realizar sobre los procesos internos y externos. A su vez, esta evaluaci n se puede medir mediante sistemas objetivos o subjetivos. Para cada uno de ellos existen pruebas y medidas $v$ lidas (Figura 1). El empleo de cada uno de estos sistemas est condicionado por la posibilidad que tengan los entrenadores para adquirir instrumentos, que suelen ser costosos. Los sistemas de monitorizaci $\mathrm{n}$ del entrenamiento son empleados cada vez m s por los entrenadores (Halson, 2014). A pesar de ello, existen pocos estudios que analicen las cargas de entrenamiento que provocan las tareas t cnico-t cticas dise adas por los entrenadores durante la semana de entrenamiento (Vargas, Urkiza \& Gil, 2015).

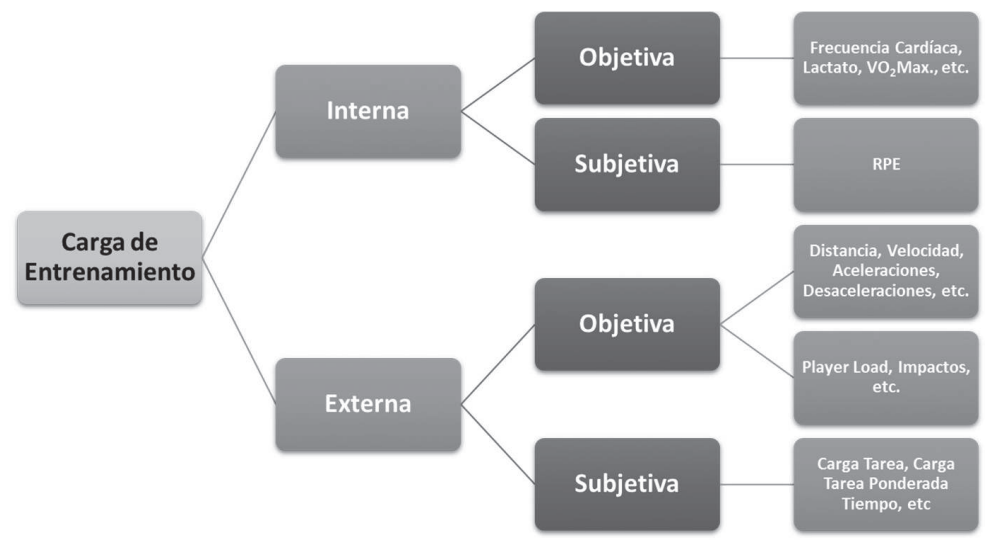

Figura 1. Sistemas de cuantificación y control de la carga de entrenamiento.

Una forma de valorar de manera no invasiva el esfuerzo realizado en el entrenamiento o competici n, se basa en las sensaciones del deportista o capacidad de control interoceptiva (Craig, 2002; Molinero, Salguero \& M rquez, 2011). Este procedimiento 
se ubica dentro del control de la carga interna del entrenamiento, y es universalmente conocido como Percepci n Subjetiva del Esfuerzo (PSE) o Rate of Perceived Exertion (RPE). La PSE es uno de los medios de control de la carga $m$ s extendidos en el entrenamiento y la competici $n$ en los deportes colectivos. Es extensa la literatura que muestra que utilizar la PSE es un $m$ todo viable para evaluar la carga de entrenamiento sin el uso de herramientas m s sofisticadas, como pueden ser los monitores de FC, pues se ha demostrado una alta correlaci n entre ambas (Castagna et al., 2011; Impellizzeri et al., 2004; Cuadrado, Chirosa, Chirosa, Mart n \& Aguilar, 2012). Aunque $m$ todos como el VO2Max, lactato en sangre o FC pueden ser $m$ s objetivos, requieren mayor formaci $n$ por parte del cuerpo t cnico (Buchheit, 2010). Las investigaciones han puesto de manifiesto la relaci $\mathrm{n}$ entre las escalas subjetivas de cuantificaci n de la carga interna, como la PSE, con medidas objetivas de carga interna, como la FC (Borresen \& Lambert, 2009; Fanchini, Azzalin, Castagna, Schena \& Impellizzeri, 2011), por lo que se propone el empleo de la PSE como medio para controlar la carga de entrenamiento, ante la ausencia recursos.

El entrenador juega un papel fundamental en el proceso de dise o del entrenamiento deportivo. Sin un proceso de planificaci n y control, es casi imposible conocer c mo se entrena de modo objetivo ( $\mathrm{lb}$ ez, 2008). La formaci n del entrenador en Espa a es un proceso reglado y estructurado que ha evolucionado muy $r$ pidamente, en aras de una uniformidad de criterios entre diferentes deportes, as como una homogenizaci $n$ de carga formativa y contenidos (Feu, Garc a, Ant nez \& lb ez, 2018). En los nuevos programas formativos del entrenador, los procesos de planificaci n y control del entrenamiento se abordan de forma espec fica con el objeto de mejorar las competencias que los entrenadores deben adquirir.

Los estudios sobre la figura del entrenador en Espa a, ponen de manifiesto que el aspecto sobre el que $\mathrm{m}$ s se investiga es acerca de su comportamiento. De forma espec fica, sobre la in- 
tervenci n del entrenador, el estilo de entrenamiento, el estilo de liderazgo o las estrategias de entrenamiento, entre otras (lb ez, Garc a, Ant nez \& Feu, 2019). Respecto a los comportamientos del entrenador, es conveniente analizar cu les son los medios y $\mathrm{m}$ todos de ense anza que actualmente llevan a la pr ctica.

Dentro de la planificaci $\mathrm{n}$ del entrenamiento, un factor de sustancial importancia es la organizaci n y estructuraci $n$ de las tareas. Diversos autores estudian la organizaci $\mathrm{n}$ de las tareas de entrenamiento (Ca adas, Rodr guez, Feu, Parejo \& Garc a, 2013). Ib ez, Feu \& Ca adas (2016), proponen un sistema integral para el an lisis de las tareas de entrenamiento (SIATE) en el que registran diferentes tipos de variables, tales como Variables Pedag gicas, Organizativas o de Carga Externa. Este tipo de iniciativas colaboran en la sistematizaci $\mathrm{n}$ del entrenamiento. La improvisaci n no puede reinar sobre la planificaci n, por muy creativo que sea el entrenador (S nchez, 1992), por lo que es necesario obtener informaci $n$ para que este pueda adaptarse a las necesidades que surjan, y ser flexible, tanto en la evoluci $n$ de los entrenamientos como en la competici n (Feu, lb ez \& Gozalo, 2007). Para valorar esta informaci n con eficacia, es necesaria una adecuada formaci $n$ acad mica y experiencia como entrenador (Feu, lb ez, Lorenzo, Jim nez \& Ca adas, 2012).

Las investigaciones han puesto de manifiesto que existe correlaci $\mathrm{n}$ entre los sistemas de cuantificaci $\mathrm{n}$ de la carga interna objetiva (medida a trav s de la FC), la carga externa objetiva (medida con dispositivos inerciales, a trav s del Player Load) y la carga externa subjetiva (medida con el SIATE, a trav s de la carga tarea ponderada al tiempo) (Reina, Mancha, Garc a, Garc a \& lb ez, 2019). Estos resultados muestran la validez de los sistemas de control de la carga mediante sistemas observacionales o subjetivos.

A trav s del SIATE se han realizado diversos estudios para cuantificar la carga externa de las tareas que utilizan los docentes de educaci $\mathrm{n} f$ sica en la ense anza de deportes de invasi $\mathrm{n}$ 
(Garc a, Feu \& lb ez, 2019; Garc a, Gamero, Gonz lez, Garc a \& Feu, 2018; Gonz lez, lb ez \& Feu, 2017), y la carga de las tareas empleadas en la formaci $n$ de jugadores en deportes de invasi n (Mancha, Garc a, Ant nez \& Garc a, 2018; Mancha, Reina, Baquero, Garc a \& lb ez, 2018), comprobando que es una herramienta til a nivel docente y en entrenamiento.

Tras un an lisis de la literatura, se aprecia la escasez de trabajos que relacionen la carga del entrenamiento con la organizaci $\mathrm{n}$ de las tareas de entrenamiento (Reina, Mancha, Feu \& Ib ez, 2017). De este problema surge la pregunta: ¿La organizaci $n$ de las tareas condiciona la carga total de las tareas de entrenamiento? En consecuencia, los objetivos espec ficos de esta investigaci n fueron: i) caracterizar el proceso de entrenamiento de un equipo a trav s del estudio de Variables Pedag gicas, Organizativas y de Carga Externa; ii) analizar las relaciones entre las Variables de Carga Externa y las Variables Pedag gicas Nivel de Oposici n y Situaci n de Juego; iii) Analizar las relaciones entre las Variables de Carga Externa y las Variables Organizativas.

\section{MÉTODO}

\section{Diseño de la investigación}

Estudio emp rico con metodolog a cuantitativa, descriptivo mediante un c digo arbitrario de observaci n, y natural porque se lleva a cabo en el contexto habitual donde se produce el fen meno y el observador no interviene en lo que observa (Montero \& Le $n, 2007$ ). Adem s, se trata de un estudio prospectivo, pues se concentra en analizar las relaciones entre las distintas variables.

\section{Participantes}

Los participantes de la investigaci $\mathrm{n}$ fueron elegidos de forma deliberada e intencional (Rodr guez, Gil \& Garc a, 1996). El equi- 
po seleccionado para el an lisis se ajust a los criterios establecidos por el equipo investigador, que fueron: nivel de experiencia del entrenador, reputaci $n$, disponibilidad y motivaci $n$ hacia el estudio. En la investigaci n particip un equipo de categor a Cadete (14/16 a os), compuesto por 13 sujetos, con altura media de $175,3 \pm 6,7 \mathrm{~cm}$ y peso medio de $64,6 \pm 19,1 \mathrm{~kg}$. El entrenador analizado contaba con gran experiencia como entrenador (26 a os), formaci n como Doctor en Ciencias de la Actividad $\mathrm{F}$ sica y del Deporte y pose a la $\mathrm{m}$ xima formaci $\mathrm{n}$ federativa. El perfil declarado del entrenador se obtuvo a trav s del Coach Intervention Questionnaire (COQ) (Feu, Ib ez, Graça \& Sampaio, 2007), en el que se aprecia el componente colaborativo, innovador y tecnol gico (Figura 2).

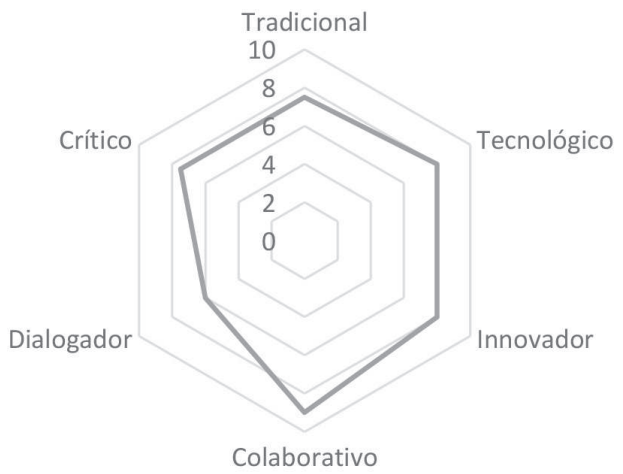

Figura 2. Perfil declarado del entrenador obtenido a través del COQ.

\section{Variables e instrumentos}

Para el estudio se consider como Variable Independiente la Carga Externa, y como Dependientes las Variables Pedag gicas y Organizativas. Las Variables Pedag gicas se seleccionaron en funci n de su influencia en la organizaci n de las tareas. Las Variables Pedag gicas del entrenamiento fueron definidas inicialmente por lb ez (2008) y posteriormente fueron complementadas por 
Ib ez et al. (2016). De las variables propuestas por estos autores, en el estudio se consider la Situaci $n$ de Juego (SI) y el Nivel de Oposici $n(N O)$. La Situaci $n$ de Juego (SJ) hace referencia a la forma de organizaci $\mathrm{n}$ de los jugadores en las tareas $1 \times 1,2 \times 2$, etc. Estas agrupaciones de jugadores pueden ser cambiantes o fijas durante toda la tarea. El sistema clasifica las tareas incrementando la complejidad en la agrupaci $\mathrm{n}$ de los deportistas. El Nivel de Oposici n (NO) hace referencia al tipo de oposici $n$ de la tarea, con un sistema de codificaci n de cinco categor as: i) sin oposici n, ii) con obst culos est ticos, iii) con obst culos din micos, iv) con oposici $n$ modulada, y v) con oposici $n$.

Para medir la Carga Externa se emplearon las variables propuestas en la escala subjetiva de Coque (2008), adaptada posteriormente por lb ez et al. (2016). Se emplearon seis variables primarias: Grado de Oposici n, Densidad de la tarea, $N$ mero de Ejecutantes Simult neos, Carga Competitiva, Espacio de Juego, e Implicaci n Cognitiva. A partir de ellas, se calculan cuatro variables secundarias, tras la transformaci $\mathrm{n}$ de los datos: Carga Total (CT), Carga Total en Segundos (CTS), Carga Ponderada (CP) y Carga Total en Minutos (CTM). La magnitud de carga de la tarea oscila en un rango de 24 valores. En la Tabla 1 se muestran los valores ponderados de Carga Total, en funci n de la categorizaci n de Carga Externa propuesta por lb ez et al. (2016).

Tabla 1. Tabla de Carga Total propuesta por lb ez et al. (2016).

\begin{tabular}{|c|c|c|c|c|c|}
\hline Ваја & & Media & Alta & & Muy Alta \\
\hline 6 & 12 & 18 & & 24 & 30 \\
\hline
\end{tabular}

Las Variables Organizativas fueron definidas inicialmente por Alarc n, C rdenas \& Ure a (2008) y posteriormente adaptadas por lb ez et al. (2016). Se emplean 4 variables primarias: Participaci n (P), Valor Participaci n (VP), Tiempo Total (TT) y Tiempo de Explicaci n (TE). De estas dos Itimas variables, se 
obtienen dos variables secundarias: Tiempo til (TU) y \% de Aprovechamiento (A). El instrumento utilizado para el an lisis fue el Sistema Integral para el An lisis de las Tareas de Entrenamiento (SIATE) (Ib ez et al., 2016).

\section{Procedimiento}

La observaci $\mathrm{n}$ y toma de datos se realiz a pie de pista. Se analizaron 38 sesiones, con un total de 340 tareas (unidades de an lisis estad stico), definidas y clasificadas bajo las mismas condiciones para el estudio, durante cuatro meses, en el segundo periodo competitivo de la temporada 2015/2016. Fueron dirigidas por el mismo cuerpo $t$ cnico.

Para el trabajo de campo y la recogida de datos se dise una hoja de observaci $n$, en la que se consignaron las variables del estudio. Los datos se registraron con los mismos materiales y por la misma persona, para garantizar que no hubiese contaminaci n externa del procedimiento. Durante las sesiones, las tareas fueron cotejadas con el entrenador, para su correcta catalogaci $n$. Para el an lisis de la informaci $n$ se realiz un filtrado de las tareas con el programa SPSS, suprimiendo aquellas que formaran parte del calentamiento, la preparaci $n f$ sica y el estiramiento. De esta forma, el $\mathrm{n}$ mero de tareas a analizar se redujo a 266. Finalmente se llev a cabo un segundo filtrado en el que se suprimieron las tareas denominadas "Tiros libres", debido a que son tareas cortas, que habitualmente se utilizan con el objetivo de que los jugadores se recuperen del trabajo de la tarea anterior, por lo que el total de tareas seleccionadas se redujo a 231 .

\section{Análisis de datos}

Se realiz un an lisis descriptivo para conocer detalladamente informaci $n$ sobre cada variable espec fica. En el an lisis descriptivo se obtuvo la frecuencia y porcentaje para variables cualitativas, y media y desviaci $n t$ pica para variables cuantitativas. 
Posteriormente se realiz un an lisis correlacional a trav $\mathrm{s}$ del coeficiente de correlaci $n$ de Pearson (variables cuantitativas) y el coeficiente de correlaci $n$ de Spearman (variables categ ricas) (Cubo, 2011). Para el an lisis estad stico se utiliz el programa inform tico SPSS 21.

\section{RESULTADOS}

Los resultados descriptivos de las Variables de Carga Externa (cuantitativas) y Variables Organizativas, tras el primer filtrado, se muestran en la tabla 2.

Tabla 2. Resultados descriptivos de Variables de Carga Externa y Variables Organizativas tras 1er filtrado.

\begin{tabular}{lccccc}
\hline & $N$ & Mínimo & Máximo & & $S D$ \\
\hline Carga Total & 266 & 7 & 26 & 20.02 & 5.24 \\
Carga Total Segundos & 266 & 1035 & 46592 & 11263.36 & 7877.18 \\
Carga Ponderada & 266 & .88 & 26.00 & 17.29 & 6.48 \\
\hline Tiempo total & 266 & 120 & 1928 & 578.09 & 291.75 \\
Tiempo Explicación & 266 & 0 & 196 & 57.50 & 39.01 \\
Tiempo útil & 266 & 115 & 1792 & 520.59 & 273.63 \\
Aprovechamiento, \% & 266 & 72.03 & 100 & 90.08 & 5.95 \\
\hline
\end{tabular}

La variable Carga Total abarca valores desde 6 hasta 30 unidades, por lo cual se puede decir que el valor medio, situado por encima de 20 unidades $(=20,02 ; S D=5,24)$, se encuentra en un rango medio-alto.

El Tiempo Total medio por tarea es de 9 minutos y 35 segundos. Sin embargo, aunque sea un valor importante para conocer c mo se entrena, habr que asegurar que durante la tarea se han conseguido los objetivos preestablecidos. En ocasiones, algunas 
tareas planteadas requieren $\mathrm{m}$ s tiempo de pr ctica que lo planeado y en otras menos, seg $\mathrm{n}$ la consecuci $\mathrm{n}$ de las metas.

El Tiempo de Explicaci $n$ medio por tarea se sit a en torno a los 60 segundos $(=57,50 ; S D=39,01)$, lo que se puede considerar un tiempo ideal en entrenamiento, pues es suficiente para que los jugadores asimilen, sin ser excesivo.

El Tiempo til para cada tarea se sit a en una media de $8 \mathrm{mi}-$ nutos 35 segundos, valor bastante ptimo en cuanto a tiempos de pr ctica en una sesi $n$, pues un menor tiempo de pr ctica podr a no generar las adaptaciones buscadas.

El Aprovechamiento del tiempo durante las tareas se sit a alrededor del $90 \%(=90,08 ; S D=5,95)$, valores bastante elevados que garantizan un tiempo de pr ctica motriz id neo para el jugador.

En la Figura 3 se muestran los resultados descriptivos de la Variable Pedag gica Situaciones de Juego en las diferentes tareas. El entrenador utiliza principalmente cuatro Situaciones de Juego. Mayoritariamente, con un 19,55\% del total, destacan las tareas de $2 \times 1$, seguida con un $18,05 \%$ por las tareas de $2 \times 0$, lo cual es normal pues es la forma de organizaci $n$ de los ejercicios de Tiros libres. Con un $17,67 \%$ y un $16,17 \%$, est $n$ las tareas de $5 \times 5$ y $1 \times 1$, respectivamente.

En la tabla 3 se muestran los resultados descriptivos de las Variables Pedag gicas y de Carga Externa, ambas cualitativas.

Tras presentar estos resultados, se realiz un segundo filtrado de datos para eliminar las tareas de Tiros libres, pues se considera que stas contaminan la muestra, y en muchos casos son utilizadas como tareas de recuperaci n. En la Tabla 4 se muestran los resultados descriptivos de las variables Carga Total y Carga Ponderada sin los ejercicios de Tiros libres $(n=231)$. Los valores de la Carga Total aumentan casi dos puntos $(21,70)$, vi ndose reducida tambi $\mathrm{n}$ la desviaci $\mathrm{n}$ t pica $(S D=3,209)$. Las tareas de entrenamiento dise adas por el entrenador, ajenas a las 


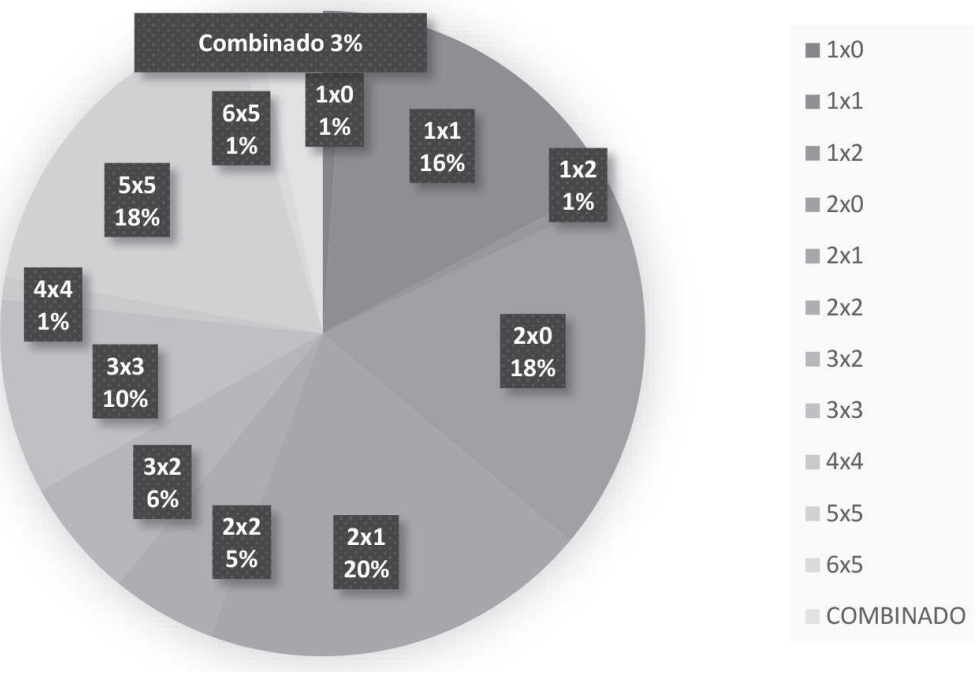

Figura 3. Resultados descriptivos de la Situación de Juego.

tareas anal ticas de lanzamiento de tiros libres, que en muchas ocasiones se utilizan para recuperaci $\mathrm{n}$ de los jugadores, tienen una carga bastante elevada. De la misma forma, los valores de Carga Ponderada tambi $\mathrm{n}$ se incrementan proporcionalmente, del 17,29 anterior, al 19,20 tras el filtrado.

La carga de las tareas es Alta/Muy Alta, como se aprecia en la Figura 4, donde se presenta la frecuencia de la Variable Carga Total. Se observa un car cter ascendente del porcentaje de cargas utilizadas, con una concentraci $\mathrm{n}$ evidente entre los valores 19 y 26. Destacan las tareas en las que la carga es 22 con un $24,68 \%$, valor similar a la media.

En la Tabla 5 se muestran los resultados correspondientes a las relaciones entre las Variables Pedag gicas Situaci $n$ de Juego y Nivel de Oposici $n$, las Variables de Carga Externa y las Variables Organizativas. 


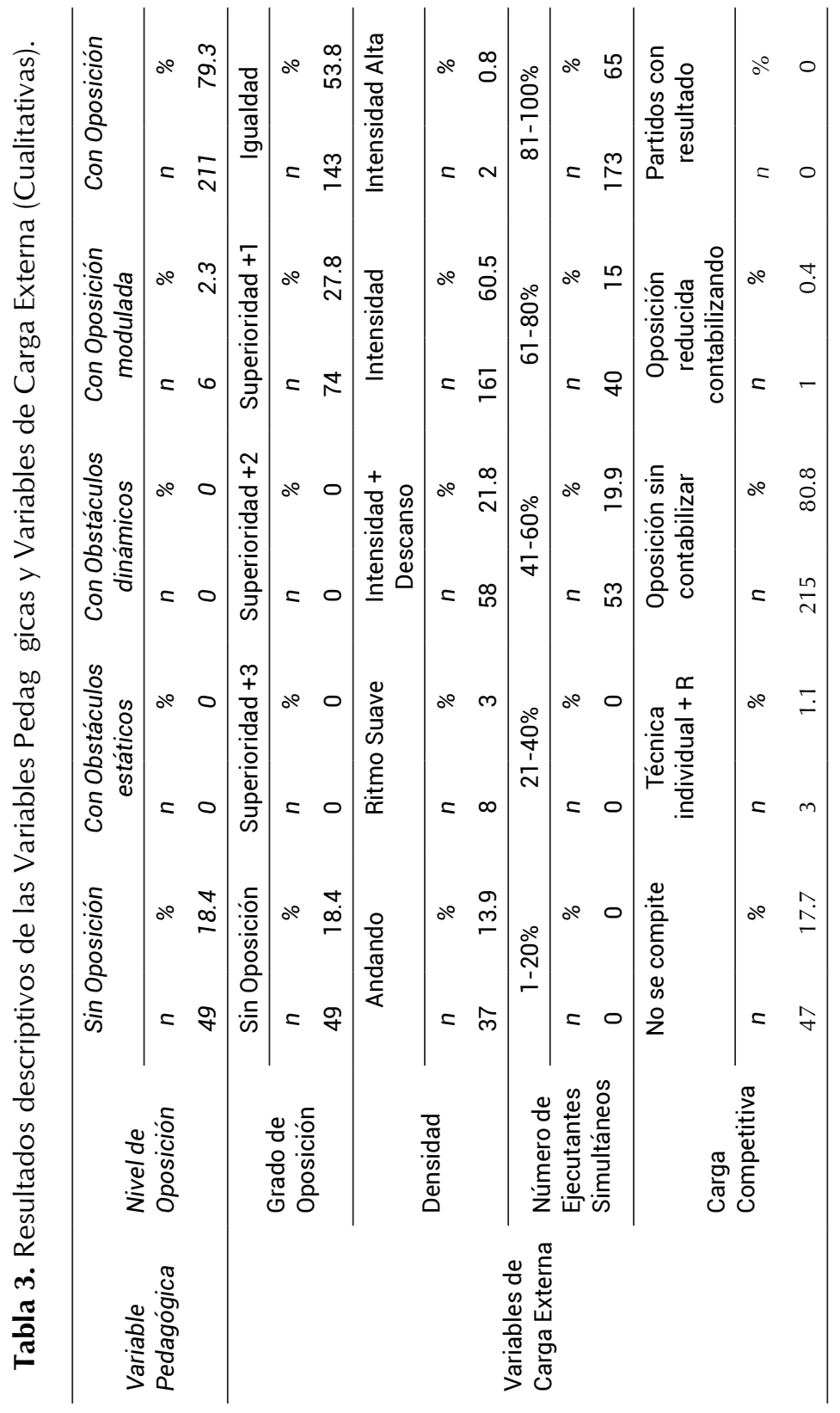

Godoy, E.; Feu, S.; Galatti, L. \& lb ez, S. J. (2017). 


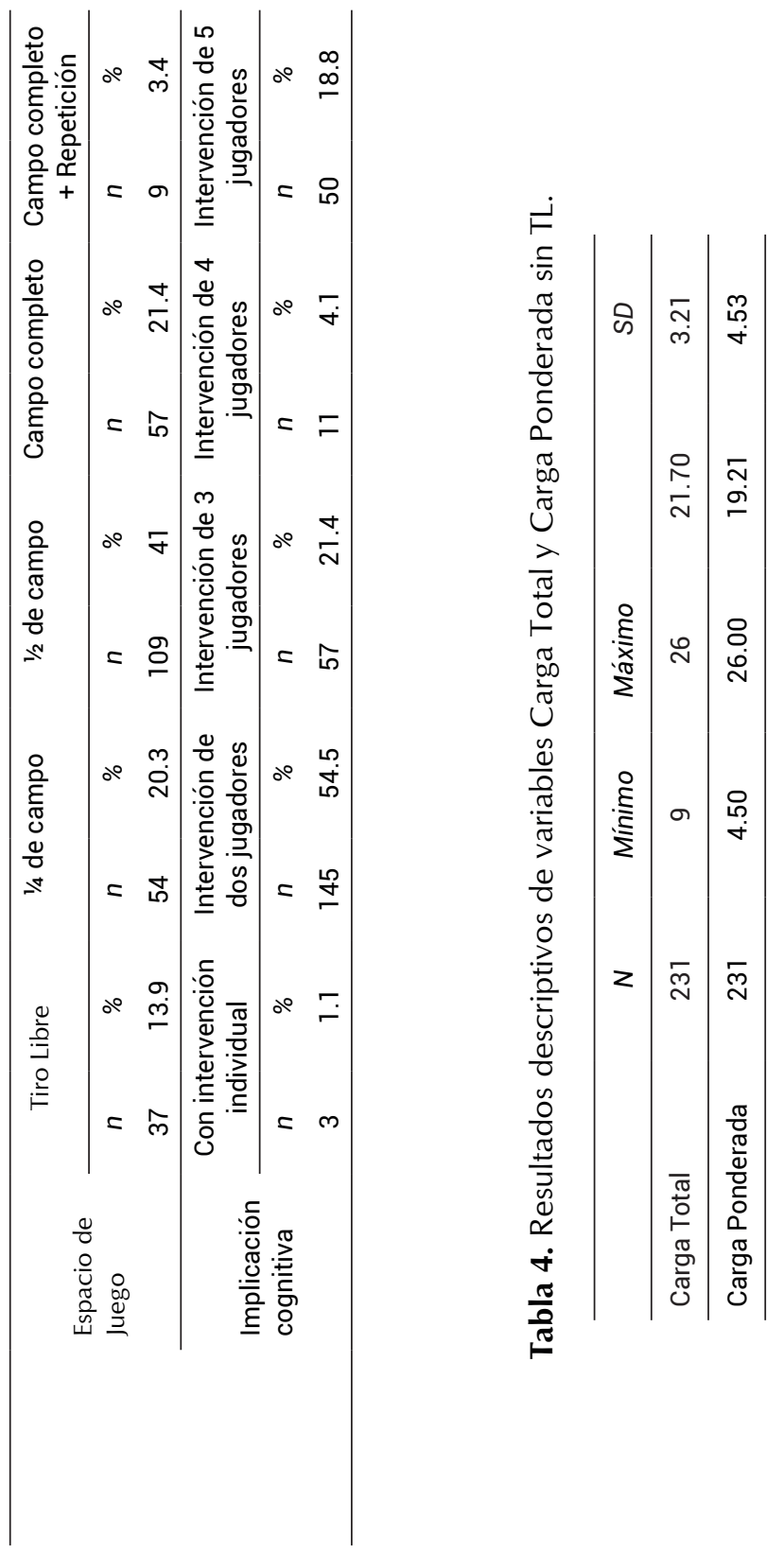




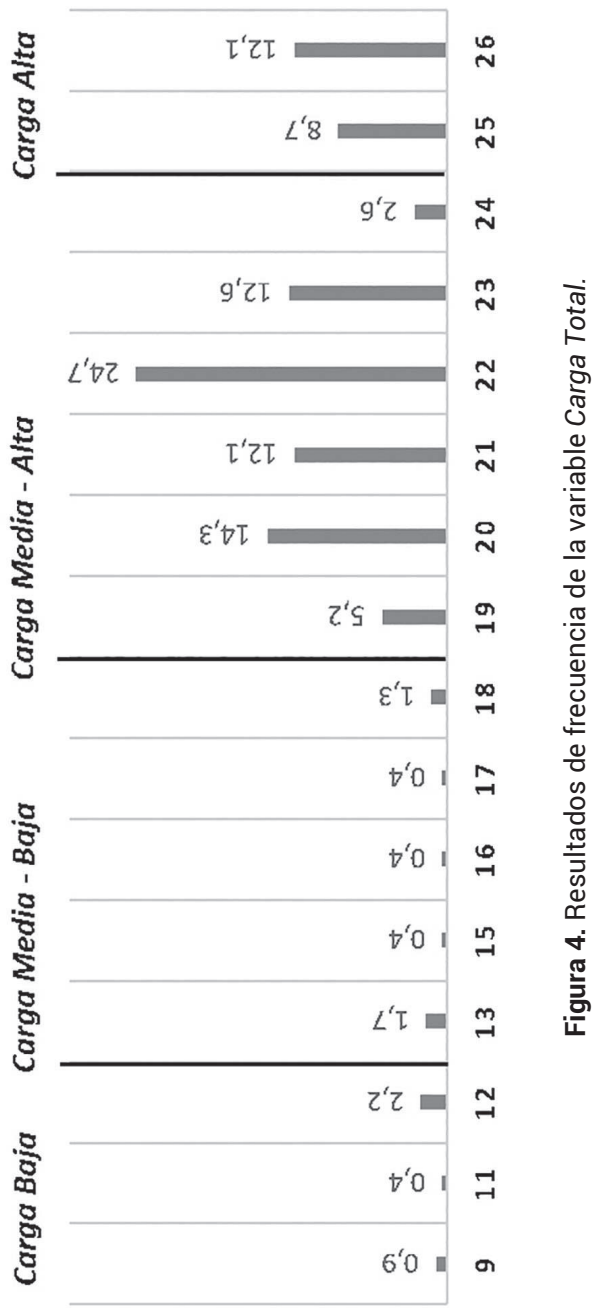

Godoy, E.; Feu, S.; Galatti, L. \& Ib ez, S. J. (2017). 


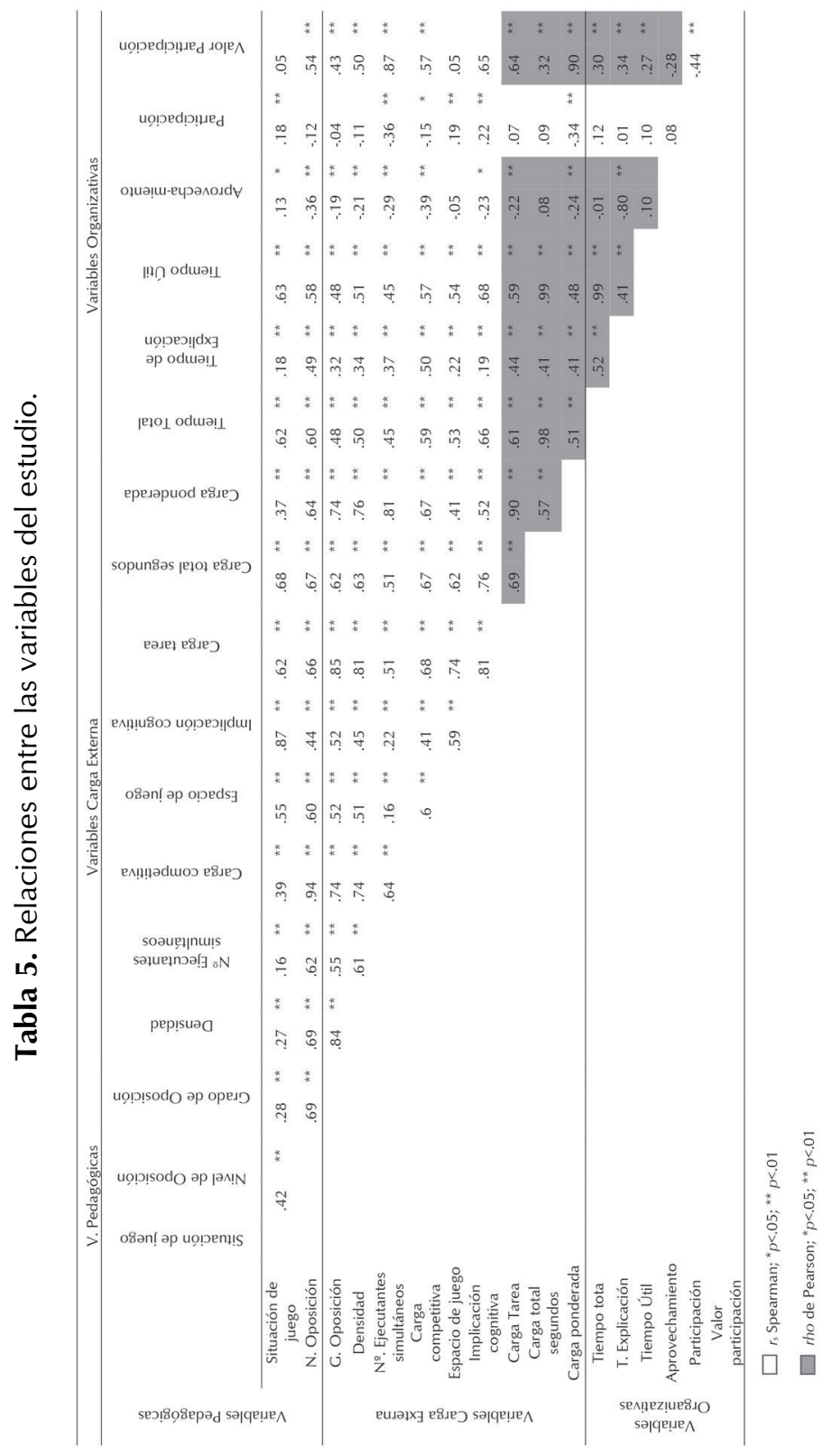

Educaci n F sica y Deporte, V. 36 (1), 
Existe un alto nivel de relaci $\mathrm{n}$ entre las variables estudiadas, mostrando que existe asociaci $n$ entre la organizaci $n$ de las tareas y la carga externa de la misma.

\section{Variables Pedagógicas}

La variable Situaci $n$ de juego mantiene una correlaci n significativa $(p<.01)$ con el resto de las variables, excepto con Aprovechamiento $(p<.05)$ y Valor Participaci $n$. Por otra parte, la variable Nivel de Oposici $n$ mantiene una correlaci n significativa $(p<.01)$ con el resto de las variables, excepto con Participaci $n$.

\section{Variables de Carga Externa}

Las variables Grado de Oposici n, № de Ejecutantes Simult neos y Carga Ponderada mantienen una correlaci $n$ significativa $(p<.01)$ con el resto de las variables. Tambi $\mathrm{n}$ Densidad y Carga Total mantienen una correlaci $n$ significativa $(p<.01)$ con el resto de las variables, excepto con Participaci $n$, con la que no existe una asociaci n. Del mismo modo, se identifica que la Carga Competitiva mantiene una relaci $\mathrm{n}$ significativa $(p<.01)$ con todas las variables, excepto con la variable Participaci $n$ $(p<.05)$. Por otra parte, la variable Espacio de Juego mantiene una correlaci n significativa $(p<.01)$ con el resto de las variables, excepto con Participaci n y Valor Participaci $n$. Se identifican en este caso similitudes con la Implicaci n Cognitiva, que tambi $n$ mantiene una correlaci $\mathrm{n}$ de $p<.01$ con todas las variables y con Valor Participaci $n(p<.05)$, salvo con la Participaci $n$. Por Itimo, la variable Carga Total Segundos mantiene una correlaci $n$ significativa $(p<.01)$ con el resto de las variables, excepto con Aprovechamiento y Participaci $n$.

\section{Variables Organizativas}

La variable Tiempo Total mantiene una correlaci $\mathrm{n}$ significativa $(p<.01)$ con el resto de las variables, excepto con Aprove- 
chamiento y Participaci n. Por otra parte, la variable Tiempo de Explicaci $n$ mantiene una correlaci $n$ significativa $(p<.01)$ con el resto de las variables, excepto con Participaci $n$. La variable Tiempo til solo mantiene correlaci $\mathrm{n}$ significativa $(p<.01)$ con Valor Participaci n, y la variable Aprovechamiento no mantiene correlaci n significativa con Participaci n ni con Valor Participaci $n$. Por Itimo, la variable Participaci $n$ mantiene una correlaci n significativa $(p<.01)$ con la variable Valor Participaci $n$.

En la Tabla 6 se muestran los resultados correlacionales de Carga Total y Carga Ponderada con las variables organizativas, tras realizar el primero y el segundo filtrado (sin tiros libres). Todas las variables correlacionan positivamente tras el primer filtrado, excepto el aprovechamiento y la participaci n. Cuando se contabilizan los tiros libres, la relaci $\mathrm{n}$ de ambas variables con la variable Aprovechamiento es negativa; sin embargo, cuando se filtran las correlaciones se convierten en positivas debido a que en las tareas de Tiros libres la Carga Total es muy reducida y el Aprovechamiento es elevado, ya que los Tiempos de Explicaci $n$ son casi inexistentes (Tiempo til muy elevado), por lo que los jugadores practican durante casi toda la tarea.

Tabla 6. Correlaciones entre la Carga Total y Carga Ponderada y las variables organizativas.

\begin{tabular}{|c|c|c|c|c|c|c|c|c|}
\hline & \multicolumn{4}{|c|}{$\begin{array}{c}\text { Con tiros libres } \\
(n=266)\end{array}$} & \multicolumn{4}{|c|}{$\begin{array}{c}\text { Sin tiros libres } \\
\quad(n=231)\end{array}$} \\
\hline & $\begin{array}{l}\text { Carga } \\
\text { Total }\end{array}$ & $p$ & $\begin{array}{c}\text { Carga } \\
\text { Ponderada }\end{array}$ & $p$ & $\begin{array}{l}\text { Carga } \\
\text { Total }\end{array}$ & $p$ & $\begin{array}{c}\text { Carga } \\
\text { Ponderada }\end{array}$ & $p$ \\
\hline Tiempo Total & .896 & ** & .505 & ** & .737 & ** & .049 & \\
\hline $\begin{array}{l}\text { Tiempo } \\
\text { Explicación }\end{array}$ & .609 & ** & .408 & ** & .501 & ** & .315 & ** \\
\hline Tiempo Útil & .436 & ** & .480 & ** & .048 & & .480 & ** \\
\hline Aprovechamiento & -.222 & ** & -.243 & ** & .314 & ** & .183 & ** \\
\hline
\end{tabular}




\begin{tabular}{llllllllll}
\hline Participación & & .073 & & -.337 & $* *$ & .157 & $*$ & -.366 & $* *$ \\
Valor & .642 & $* *$ & .902 & $* *$ & .281 & $* *$ & .846 & $* *$ \\
Participación & & & & & & & & & \\
\hline
\end{tabular}

** $p<.01 ; * p<.05 /$

(+) En la variable Participación se empleó el coeficiente de correlación de Spearman.

\section{DISCUSIÓN}

El objetivo de la investigaci $\mathrm{n}$ fue identificar si la organizaci $\mathrm{n}$ de las tareas de entrenamiento condiciona la carga de entrenamiento. Los resultados ponen de manifiesto $\mathrm{m}$ Itiples relaciones entre las variables que ayudan a definir una tarea de entrenamiento a nivel organizativo y la carga subjetiva que soportan los deportistas. El equipo analizado se caracteriza por un predominio de las tareas con oposici $n$ activa, utilizaci $n$ de $1 / 2$ de pista, con una participaci $\mathrm{n}$ alta, con una carga de la tarea media-alta y elevados niveles en los tiempos de pr ctica motriz por parte de los jugadores, con reducidos tiempos de explicaci n. La Carga Externa de las tareas es elevada cuando el entrenador utiliza tareas con oposici n (Nivel de Oposici n) y la Situaci $n$ de Juego tiende hacia un elevado $n$ mero de participantes que act an en oposici n. Adem s, la Situaci $n$ de Juego influye tambi $n$ en el Nivel de Oposici n, pues cuanto mayor sea el valor de sta, mayor Nivel de Oposici n existir , y viceversa. La Carga Externa de las tareas ser m s elevada cuando las Variables Organizativas Tiempo Total, Tiempo de Explicaci n, Tiempo til y Valor Participaci $n$ sean mayores, y menos elevada cuando el Aprovechamiento sea alternativo o consecutivo.

La informaci $\mathrm{n}$ sobre las tareas de entrenamiento ayuda al entrenador deportivo al control del proceso de entrenamiento (Ca adas \& lb ez, 2010; lb ez et al., 2009). Por ello se ha llevado a cabo un an lisis de las Variables Pedag gicas Situaci $n$ 
de Juego y Nivel de Oposici n, las Variables Organizativas y las Variables de Carga Externa.

El Aprovechamiento del tiempo durante las tareas fue muy elevado, pues supera el rango establecido como ptimo, entre $70 \%$ y $80 \%$ del tiempo total (Alarc n et al., 2008; Pi ron, 1988). El Aprovechamiento del tiempo es 10 puntos superior al reportado por Alarc n et al. (2008), en su estudio con deportistas de categor a Cadete. Igualmente, estos autores proponen que el tiempo dedicado a la organizaci n y explicaci n inicial, debe oscilar entre $10 \%$ y $20 \%$ del tiempo total. Los valores obtenidos en este estudio sit an el tiempo de explicaci n en un 10,05\% de media para cada tarea, valor ptimo seg $\mathrm{n}$ los indicadores de referencia.

En relaci n con el Nivel de Oposici $n$, se han utilizado m s de un $80 \%$ de tareas con oposici n. Ortega, C rdenas, Sainz \& Palao (2006) identificaron que la mayor parte de los lanzamientos que se realizan en un partido de baloncesto se producen con oposici n. Cuanto mayor es el nivel de pericia de los jugadores, stos buscan situaciones de lanzamiento con menor oposici n (Ib ez, Feu, Garc a, Parejo \& Ca adas, 2009), por lo que realizar situaciones de entrenamiento con oposici n colabora en la formaci $\mathrm{n}$ del jugador. Para entrenar de forma contextualizada (aprendizaje situado), es necesario que las condiciones de la pr ctica sean lo m s parecidas a la realidad (Ca adas, Parejo, Ib ez, Garc a \& Feu, 2009). Adem s, Gim nez \& S enz (2007) y $S$ enz (2009), a aden que el trabajo con oposici $n$ favorece el aprendizaje, no solo por el hecho de suponer un reto, sino tambi $\mathrm{n}$ al generar incertidumbre, lo que permite que el jugador desarrolle la percepci $\mathrm{n}$ y la toma de decisiones inherentes a este deporte.

Otro rasgo a destacar, es que en un $61 \%$ de las tareas analizadas el $n$ mero de participantes simult neos oscila entre $81 \%$ y $100 \%$. La participaci n simult nea de jugadores refrenda que el planteamiento metodol gico se aproxima a un modelo que 
facilita la participaci $\mathrm{n}$ activa del deportista en su aprendizaje y comprensi n del juego (Ib ez, Jim nez \& Ant nez, 2015). A jugar se aprende jugando, por lo que el incremento de la pr ctica, reduciendo los tiempos de espera, mejora los aprendizajes.

Con respecto a la variable Situaci $n$ de Juego, en categor as de formaci n, Graça \& Oliveira (1997) y C rdenas (2006) manifiestan su predilecci $\mathrm{n}$ por una situaci $\mathrm{n}$ de juego colectiva reducida de $3 \times 3$, frente a una situaci $\mathrm{n}$ de $5 \times 5$. Para la categor a cadete, C rdenas (2006) contempla la importancia de una sucesiva incorporaci $\mathrm{n}$ del $5 \times 5$, pues en categor as anteriores se marca la necesidad de potenciar el juego colectivo a trav $\mathrm{s}$ de situaciones de juego reducido. Para lb ez (2008) y Gim nez (2009), la aplicaci n de una metodolog a constructivista del aprendizaje implica una utilizaci $\mathrm{n}$ progresiva de situaciones de juego, de simples $(1 \times 1)$ a complejas $(2 \times 2,3 \times 3,4 \times 4)$. Esta progresi $\mathrm{n}$ debe ser aplicada en la sesi $\mathrm{n}$ de entrenamiento, en cada microciclo, en todos los per odos de una temporada y durante toda la etapa formativa del jugador de baloncesto. El entrenador que particip en el presente estudio, prefiri situaciones $1 \times 1$ y $2 \times 1$, pues se trata de una categor a de formaci $n, y$ dio relevancia a situaciones $5 \times 5$, pues un modelo de aprendizaje que pretende que sus aprendices comprendan la complejidad y la utilidad de lo que hacen en el juego, requiere de situaciones de aprendizaje pr ximas al juego real (Ortega \& Victoria, 2015).

Con relaci $\mathrm{n}$ a la carga de entrenamiento, Foster et al. (2001) afirman que la medici $\mathrm{n}$ mediante frecuencia cardiaca de ejercicios de intensidad muy alta no es objetiva, y sugieren que es mucho $\mathrm{m} \mathrm{s}$ til emplear $\mathrm{m}$ todos subjetivos de medici $n$. Reina, Mancha, Garc a \& Ib ez (2017) identificaron una alta correlaci $n$ entre la carga objetiva medida a trav s de dispositivos inerciales, con la carga subjetiva medida a trav s del instrumento SIATE, empleado en este estudio. Otras investigaciones miden la carga externa con $\mathrm{m}$ todos como la PSE, utilizando la escala de Borg en sus distintas variantes (Coutts et al., 2008; Alexiou \& 
Coutts, 2008; Cuadrado et al., 2012; Scott et al., 2013; Feu, Carrillo, Fuentes, Refoyo \& Calleja, 2015), por su f cil aplicaci n y disponibilidad (Utter et al., 2007). La Carga Total de entrenamiento de las tareas analizadas es media-alta, con 20,02 unidades, superando el valor medio propuesto por lb ez et al. (2016).

Las Variables Pedag gicas Situaci $n$ de Juego y Nivel de Oposici $n$ mantienen una relaci $n$ significativa con el resto de las variables. Cuanto $\mathrm{m}$ s se aproxima la situaci $\mathrm{n}$ de juego a la realidad (5x5) mayor es la carga de la tarea. Adem s, el mayor valor de Participaci n (consecutiva), se produce por un incremento de la asociaci $\mathrm{n}$ de los jugadores.

La Situaci $n$ de Juego dispuesta por el entrenador en la tarea, influir en la forma de participaci $\mathrm{n}$ en la misma. Adem s, mantiene una relaci $\mathrm{n}$ significativa tambi $\mathrm{n}$ positiva con las de$\mathrm{m}$ s variables, excepto con Aprovechamiento. Esto se debe a que las tareas con Situaci $n$ de Juego reducida $(2 \times 2,2 \times 1,1 \times 1)$ tienen un gran Aprovechamiento, pues el Tiempo til es elevado al tratarse de tareas muy b sicas del juego y de $f$ cil agrupaci n. En equipos de base, los entrenadores prefieren situaciones de juego reducido (Ca adas, Ib ez, Garc a, Parejo \& Feu, 2013), siendo $\mathrm{m}$ s exigentes conforme disminuye el $\mathrm{n}$ mero de participantes, constat ndose en el aumento de la frecuencia cardiaca (Gracia, Garc a, Ca adas \& Ib ez, 2014; Ortega, Palao \& Puigcerver, 2009). Una correcta organizaci n de las tareas de entrenamiento mediante situaciones reducidas (Small Side Games), empleando todo el terreno de juego, en la que todos los jugadores participen de forma simult nea, incrementa el tiempo til y la carga de entrenamiento.

El Nivel de Oposici $n$ tambi n condiciona la carga de la tarea, correlaciona positivamente con cada una de las categor as que la definen y, por lo tanto, la alteraci $\mathrm{n}$ de esta variable va a producir variaciones en la carga del entrenamiento. Su correlaci n con la Carga Total es elevada. Adem s, estas dos variables correlacionan entre $s$ de forma positiva. Las tareas con oposi- 
ci $n$, y en relaci $n$ con ello las situaciones de $3 \times 3$, se ha constatado en la literatura que son las que provocan demandas $\mathrm{m} \mathrm{s}$ altas en los jugadores (Coutinho et al., 2016) y por tanto mejoran el rendimiento f sico (Katis \& Kellis, 2009). El dise o de tareas con oposici $\mathrm{n}$ en deportes de invasi $\mathrm{n}$ incrementa la carga que soportan los deportistas.

Tambi n existe una correlaci n con las Variables Organizativas, aunque el Nivel de Oposici n no mantiene correlaci n con la variable organizativa Participaci $n$, variable categ rica que define si una tarea es de ejecuci $n$ simult nea, alternativa o consecutiva. Se entiende que son variables independientes, pues una tarea puede ser de organizaci n simult nea, alternativa o consecutiva, independientemente del nivel de oposici $n$ de esta. Las Variables Organizativas tambi n muestran correlaciones generalizadas con las Variables de Carga Externa, por lo que se puede plantear que la organizaci $\mathrm{n}$ del entrenamiento condiciona la carga.

Cabe decir que la variable Carga Total es fruto de la suma de las seis variables de las que se obtiene su valor. Por lo tanto, se puede entender que las relaciones con las seis variables primarias que la originan es I gica. No obstante, existe un factor contaminante en el estudio que deriva de las tareas de Tiros libres y Lanzamientos anal ticos por parejas. Estas tareas de carga reducida y gran aprovechamiento, implican que la relaci $n$ entre las variables Carga Total o Carga Ponderada con la variable Aprovechamiento sea negativa, aunque esto se invierte cuando se eliminan las tareas de tiros libres.

Entre las Variables Organizativas tambi n existen relaciones, de las que destacan la relaci $\mathrm{n}$ negativa entre Tiempo Explicaci $n$ y Aprovechamiento. Esto se debe a que, cuanto $\mathrm{m}$ s tiempo se dedica a explicar, menos provecho se sacar de la tarea, y menos tiempo de pr ctica motriz tendr el jugador. Es necesario buscar organizaciones eficaces que permitan disminuir el tiempo de explicaci n y la optimizaci $n$ del tiempo til (Alarc $n$ et 
al., 2008). Adem s, la correlaci $n$ entre Participaci $n$ y Valor Participaci $n$ tambi $n$ fue negativa. El sistema de categor as ayuda a interpretar estos resultados. Un valor bajo en Participaci $n$ (simult nea) se relaciona con un Valor Participaci $n$ alto (cociente entre asistentes y participantes alto).

\section{CONCLUSIONES}

El conocimiento de la estructura de las tareas de entrenamiento a trav s de las variables pedag gicas y organizativas, permite identificar la carga subjetiva de las tareas $y$, por extensi $n$, de la sesi $\mathrm{n}$ de entrenamiento. La modificaci $\mathrm{n}$ de los factores pedag gicos y organizativos de la tarea implica una alteraci $n$ de la carga de entrenamiento. Los entrenadores deben conocer y usar esta informaci n para modular el proceso de entrenamiento, incrementando los niveles de desarrollo de sus deportistas. Por ello es recomendable priorizar el uso de situaciones de juego reducido (Small Side Games), con un n mero bajo de jugadores $(2 \times 2$ y $3 \times 3)$, adem $\mathrm{s}$ del necesario juego individual $(1 \times 1)$ para aumentar la carga competitiva y la implicaci n cognitiva, variables especialmente relacionadas con la carga externa de la tarea. Adem s, es necesario prestar especial atenci $n$ a la organizaci $\mathrm{n}$ de la tarea, para favorecer la participaci $\mathrm{n}$ simult nea en un alto porcentaje de jugadores, con una adecuada gesti $n$ del tiempo para aumentar el tiempo til de la tarea. Finalmente, el entrenador podr utilizar el feedback intencionalmente para aumentar la intensidad cuando sea necesario. Por todo ello, antes de poner en pr ctica sus sesiones de entrenamiento, los entrenadores pueden analizar las tareas de aprendizaje a trav $\mathrm{s}$ del SIATE, pues proporciona informaci $n$ til $y v$ lida sobre las diferentes variables que definen una tarea optimizando el proceso de entrenamiento. 


\section{REFERENCIAS}

1. Akubat, I., \& Abt, G. (2011). Intermittent exercise alters the heart rate-blood lactate relationship used for calculating the training impulse (TRIMP) in team sport players. Journal of Science and Medicine in Sport, 19, 249-253.

2. Alarc n, F., C rdenas, D., \& Ure a, N. (2008). Influencia de los factores de organizaci $\mathrm{n}$ de las tareas de aprendizaje sobre los tiempos de pr ctica del jugador de baloncesto. Apunts, Educaci n F sica y Deportes, 92, 46-55.

3. Alexiou, H., \& Coutts, A. (2008). A comparison of methods used for quantifying internal training load in women soccer players. International Journal of Sports Physiology and Performance, 3, 320-330.

4. Borresen, J., \& Lambert, M. (2009). The quantification of training load, the training response and the effect on performance. Sports Medicine, 39(9), 779-795.

5. Buchheit, M. (2010). Performance and physiological responses to repeated-sprint and jump sequences. European Journal of Applied Physiology, 110(5), 1007-1018.

6. Ca adas, M., \& Ib ez, S. (2010). La planificaci $n$ de contenidos de entrenamiento de baloncesto en equipos de iniciaci n. E-Balonmano. com. Revista de Ciencias del Deporte, 6(1), 49-65.

7. Ca adas, M., Ib ez, S., Garc a, J., Parejo, I., \& Feu, S. (2013). Las situaciones de juego en el entrenamiento de baloncesto en categor as base. Revista Internacional de Medicina y Ciencias de la Actividad F sica y del Deporte, 13(49), 41-54.

8. Ca adas, M., lb ez, S., Feu, S., Garc a, J., \& Parejo, I. (2011). An lisis de los medios de entrenamiento en un equipo de Minibasket y la influencia de un programa formativo para el entrenador. Un estudio de caso. gora para la Educaci n F sica y el Deporte, 13(3), 363-382.

9. Ca adas, M., Parejo, I., lb ez, S., Garc a, J., \& Feu, S. (2009). Relaci n entre las variables pedag gicas de entrenamiento en un equipo de Minibasket. Cuadernos de Psicolog a del Deporte, 9(Supl.), 50.

10. Ca adas, M., Rodr guez, G., Feu, S., Parejo, I., \& Garc a, J. (2013). Relathionship between pedagogical content knowledge and coaching methods. Revista de Psicolog a del Deporte, 22(1), 183-186.

11. C rdenas, D. (2006). El proceso de formaci n t ctica colectiva en el baloncesto desde la perspectiva constructivista. EF Deportes Revista Digital, 94, 1-15.

12. Casamichana, D., Castellano, J., Calleja, J., San Rom n, J., \& Castagna, C. (2013). Relationship between indicators of training load in soccer players. The Journal of Strength \& Conditioning Research, 27, 369-374. 
13. Castagna, C., Impellizzeri, F., Chaouachi, A., Bordon, C., \& Manzi, V. (2011). Effect of training intensity distribution on aerobic fitness variables in elite soccer players: a case study. The Journal of Strength \& Conditioning Research, 25(1), 66-71.

14. Coque, I. (2008). Valoraci n subjetiva de la carga del entrenamiento t cnico-t ctico. Una aplicaci n pr ctica (I). Clinic, 81, 39-43.

15. Coque, I. (2009). Valoraci n subjetiva de la carga del entrenamiento t cnico-t ctico. Una aplicaci n pr ctica (II). Clinic, 82, 42-45.

16. Coutinho, D., Reis, S., Gonçalves, B., Pereira, A., Sampaio, A., \& Leite, N. (2016). Manipulating the number of players and targets in team sports. Small-Sided Games during Physical Education classes. Revista de Psicolog a del Deporte, 25(1), 169-177.

17. Coutts, A., Rampinini, E., Marcora, S., Castagna, C., \& Impellizzeri, F. (2008). Heart rate and blood lactate correlates of perceived exertion during small-sided soccer games. Journal of Science and Medicine in Sport, 12(1), 79-84.

18. Craig, A. (2002). How do you feel? Interoception: The sense of the physiological condition of the body. Nature Reviews Neuroscience, 3, 655-666.

19. Cuadrado, J., Chirosa, L., Chirosa, I., Mart n, I., \& Aguilar, D. (2012). La percepci n subjetiva del esfuerzo para el control de la carga de entrenamiento en una temporada en un equipo de balonmano. Revista de Psicolog a del Deporte, 21(2), 331-339.

20. Cubo, S. (2011). La investigaci n experimental. En S. Cubo, B. Mar n \& J. Ramos (Eds). $M$ todos de investigaci $n$ y an lisis de datos en ciencias sociales y de la salud (pp.235-326). Madrid: Ediciones Pir mide.

21. Dehesa, R., Vaquera, A., Garc a, J., \& Bay n, P. (2015). Hearth rate analysis of high level basketball players during training sessions. Revista de Psicolog a del Deporte, 24, 17-19.

22. Echeverri, J. (2012). Algunas consideraciones sobres los deportes de cooperaci n y oposici n. VIREF Revista de Educaci n F sica, 1(1), 70-78.

23. Fanchini, M., Azzalin, A., Castagna, C., Schena, F., \& Impellizzeri, F. (2011). Effect of bout duration on exercise intensity and technical performance of small-sided games in soccer. The Journal of Strength \& Conditioning Research, 25(2), 453-458.

24. Ferreira, A., Ib ez, S., \& Sampaio, J. (2009). Las reglas y la casualidad en Baloncesto: una aproximaci $\mathrm{n}$ hist rica. Retos. Nuevas Tendencias en Educaci n F sica, Deporte y Recreaci n, 15, 9-13.

25. Feu, S., Carrillo, A., Fuentes, M., Refoyo, I., \& Calleja, J. (2015). Perception of effort in minibasketball during small side games. Revista de Psicolog a del Deporte, 24, 21-25. 
26. Feu, S., Garc a, J., Ant nez, A., \& Ib ez, S. (2018). Coaching and coach education in Spain: A critical analysis of legislative evolution. International Sport Coaching Journal, 5(3), 281-292.

27. Feu, S., Ib ez, S., Lorenzo, A., Jim nez, S., \& Ca adas, M. (2012). El conocimiento profesional adquirido por el entrenador de balonmano: experiencias y formaci n. Revista de Psicolog a del Deporte, 21(1), 107-115.

28. Feu, S., Ib ez, S., \& Gozalo, M. (2007). Propiedades psicom tricas de los cuestionarios EDD y EPD para evaluar el estilo de planificaci $n$ y decisi $n$ de los entrenadores. Revista de Psicolog a del Deporte, 16(2), 185-199.

29. Feu, S., Ib ez, S., Graça., A., \& Sampaio, J. (2007). Evaluaci n psicom trica del cuestionario de orientaci $\mathrm{n}$ de los entrenadores en una muestra de entrenadores espa oles de balonmano. Psicothema, 19(4), 698-704.

30. Foster, C., Florhaug, J., Franklin, J., Gottschall, L., Hrovatin, L., Parker, S., \& Dodge, C. (2001). A new approach to monitoring exercise training. Journal of Strength and Conditioning Research, 15(1), 109-115.

31. Garc a, J., Feu, S., \& Ib ez, S. 2019). Comparative study of two intervention programmes for teaching soccer to school-age students. Sports, 7(3), 74.

32. Garc a, J., Gamero, M, Gonz lez, S., Garc a, J., \& Feu, S. (2018). Study of the external training load of tasks for the teaching of handball in preservice teachers according to their genre. E-Balonmano.com: Revista de Ciencias del Deporte, 14(1), 45-54.

33. Gim nez, F. (2009). El dise o de la sesi $n$ en la iniciaci $n$ al baloncesto. En G. Ortega \& A. Jim nez (Eds.), T ctica y $t$ cnica en la iniciaci $n$ al baloncesto (pp.135-145). Sevilla: Wanceulen.

34. Gim nez, F., \& S enz, P. (2007). Estrategias I dicas para mejorar la percepci $n$ y decisi $n$ de los jugadores y jugadoras. En G. Ortega \& A. Jim nez (Coords.), Baloncesto en la iniciaci $n$ (pp. 123-138). Sevilla: Wanceulen.

35. G mez, C., Gamonales, J., Pino, J., \& lb ez, S. (2018). Comparative analysis of load profile between small-sided games and official matches in youth soccer players. Sports, 6(4), 173.

36. Gonz lez, S., lb ez, S., \& Feu, S. (2017). Dise o de dos programas de ense anza del baloncesto basados en dos $m$ todos de ense anzaaprendizaje diferentes. E-Balonmano.com: Revista de Ciencias del Deporte, 13(2), 131-152.

37. Graça, A., \& Oliveira, J. (1997). La ense anza de los juegos deportivos. Barcelona: Paidotribo.

38. Gracia, F., Garc a, J., Ca adas, M., \& lb ez, S. (2014). Diferencias en la frecuencia card aca en situaciones de juego modificadas en balon- 
cesto de formaci n. E-Balonmano.com: Revista de Ciencias del Deporte, 10(1), 23-30.

39. Halson, S. (2014). Monitoring training load to understand fatigue in athletes. Sports Medicine, 44(2), 139-147.

40. Hern ndez, J. (1994). Fundamentos del deporte. An lisis de las estructuras del Juego Deportivo. Barcelona: INDE.

41. Hoyos, G. (2014). M todo pedagog a de las situaciones: su influencia en la toma de decisiones de la fase de ataque de jugadoras de baloncesto. Educaci n F sica y Deporte, 33(1), 107-127.

42. Ib ez, S. (2008). La planificaci $\mathrm{n}$ y el control del entrenamiento t cnico-t ctico en baloncesto. En N. Terrados \& J. Calleja (Coords.), Fisiolo$\mathrm{g}$ a, entrenamiento y medicina del baloncesto (pp. 299-313). Barcelona: Paidotribo.

43. Ib ez, S., Feu, S., Garc a, J., Parejo, I., \& Ca adas, M. (2009). Shot differences between professional (ACB) and amateur (EBA) basketball teams. Multifactorial study. Revista de Psicolog a del Deporte 18(Supl.), 313-317.

44. Ib ez, S., Feu, S., \& Ca adas, M. (2016). Sistema Integral Para el An lisis de las tareas de Entrenamiento, SIATE, Deportes de Invasi n. EBalonmano.com: Revista de Ciencias del Deporte, 12(1), 3-30.

45. Ib ez, S., Garc a, J., Ant nez, A., \& Feu, S. (2019). Coaching in Spain research on the sport coach in Spain: A systematic review of doctoral theses. International Sport Coaching Journal, 6(1), 110-125.

46. Ib ez, S., Jim nez, A., \& Ant nez, A. (2015). Differences in basketball training loads between comprehensive and technical models of teaching/training. Revista de Psicolog a del Deporte, 24, 47-50.

47. Impellizzeri, F., Rampinini, E., Coutts, A., Sassi, A., \& Marcora, S. (2004). Use of RPE-based training load in soccer. Medicine and Science in Sports and Exercise, 36(6), 1042-1047.

48. Katis, A., \& Kellis, E. (2009). Effects of small-sided games on physical conditioning and performance in young soccer players. Journal of Sports Science and Medicine, 8(3), 374-380.

49. Mancha, D., Garc a, J., Ant nez, A., \& Garc a, J. (2018). ¿Afecta la fase de juego al dise o de las tareas de un equipo de baloncesto de formaci n? Sport-TK: Revista Euroamericana de Ciencias del Deporte, 7(2), 27-36.

50. Mancha, D., Reina, M., Baquero, B., Garc a, J., \& lb ez, S. (2018). An lisis de carga competitiva en jugadores de balonmano de formaci $\mathrm{n}$ en funci $\mathrm{n}$ del resultado final. E-Balonmano.com: Revista de Ciencias del Deporte, 14(2), 99-108.

51. Molinero, O., Salguero, A., \& M rquez, S. (2011). An lisis de la recuperaci $\mathrm{n}$-estr $\mathrm{s}$ en deportistas y relaci $\mathrm{n}$ con los estados de 
nimo: un estudio descriptivo. Cuadernos de Psicolog a del Deporte, 11(2), 47-55.

52. Montero, I., \& Le n, O. (2007). A guide for naming research studies in Psychology. International Journal of Clinical and Health Psychology, 7(3), 847-862.

53. Ortega, E., C rdenas, D., S inz, P., \& Palao J. (2006). Analysis the final action used in basketball during formative years according to player's position. Journal of Human Movements Studies, 50(4), 421- 437.

54. Ortega, E., Palao, J., \& Puigcerver, C. (2009). Frecuencia cardiaca, formas de organizaci $\mathrm{n}$ y situaciones de juego en baloncesto. Revista Internacional de Medicina y Ciencias de la Actividad F sica y del Deporte, 9(36), 393-413.

55. Ortega, T., \& Victoria, S. (2015). Caracter sticas del contraataque en baloncesto de formaci n. Educaci n F sica y Deporte, 34 (2), 429-465.

56. Pascual, N., Llorca, V., Carbonell, J., \& P rez, J. (2016). An lisis de la carga interna en los entrenamientos de $f$ tbol sala femenino de $1^{\text {a }}$ divisi n nacional. Sportis. Scientific Technical Journal of Sport, Physical Education and Psychomotricity, 2(2), 254-267.

57. Paulson, T., Mason, B., Rhodes, J., \& Goosey, V. (2015). Individualized internal and external training load relationships in elite wheelchair rugby players. Frontiers in Physiology, 6, 388.

58. Pi ron, M. (1988). Did ctica de las actividades $f$ sicas y deportivas. Madrid: Gymnos.

59. Reina, M., Mancha, D., Feu, S., \& lb ez, S. (2017) ¿Se entrena como se compite? An lisis de la carga en baloncesto femenino. Revista de Psicolog a del Deporte, 26(1), 9-13.

60. Reina, M., Mancha, D., Garc a, D., \& Ib ez, S. (2017). An lisis correlacional de la carga objetiva y subjetiva de entrenamiento en baloncesto femenino. En 13 Congreso internacional de Ciencias del Deporte y la Salud, Medicina, enfermer a, Fisioterapia, Psicolog a, Nutrici n, educaci $n$ F sica y Deporte. Pontevedra (Espa a).

61. Reina, M., Mancha, D., Garc a, D., Garc a, J., \& Ib ez, S. (2019). Comparaci $\mathrm{n}$ de tres $\mathrm{m}$ todos de cuantificaci $\mathrm{n}$ de la carga de entrenamiento en baloncesto. RICYDE. Revista Internacional de Ciencias del Deporte, 15(58), 368-382.

62. Rodr guez, G., Gil, J., \& Garc a, E. (1996). M todos de investigaci n cualitativa. M laga: Aljibe.

63. S enz, P. (2009). Dise o de tareas t cticas y t cnicas en la iniciaci $\mathrm{n}$ al baloncesto. En G. Ortega \& A. Jim nez (Coords.), T ctica y $t$ cnica en la iniciaci $n$ al baloncesto (pp.117-133). Sevilla: Wanceulen.

64. Sampaio, J., Abrantes, C., \& Leite, N. (2009). Power, heart rate and perceived exertion responses to $3 \times 3$ and $4 \times 4$ basketball small-sided. Revista de Psicolog a del Deporte, 18, 463-467. 
65. S nchez, F. (1992). Bases para una did ctica de la educaci $n$ f sica y el deporte. Madrid: Gymnos.

66. S nchez, M. (2007). El acondicionamiento $\mathrm{f}$ sico en baloncesto. Apunts Medicine de l'Esport, 42(154), 99-107.

67. Scott, B., Lockie, R., Knight, T., Clark, A., \& Janse, X. (2013). A comparison of methods to quantify the in-season training load of professional soccer players. International Journal of Sports Physiology and Performance, 8, 195-202.

68. Tapia, A., \& Hern ndez, A. (2011). Una herramienta para estudiar la direcci $\mathrm{n}$ de equipos en $\mathrm{f}$ tbol. Cuadernos de Psicolog a del Deporte, 11(2), 105-113.

69. Utter, A., Kang, J., Nieman, D., Dumke, C., McAnulty, S., \& McAnulty, L. (2007). Ratings of perceived exertion during intermittent and continuous exercise. Perceptual and Motor Skills, 104, 1079-1087.

70. Vargas, A., Urkiza, I., \& Gil, S. (2015). Efecto de los partidos de pretemporada en la planificaci $\mathrm{n}$ deportiva: Variabilidad en las sesiones de entrenamiento. Retos. Nuevas Tendencias en Educaci n F sica, Deporte y Recreaci $n, 27,45-51$. 